ERNO will be completed early in 1974. Award of the development contract is scheduled for mid-1974. Spacelab will comprise a pressurised laboratory providing a shirt-sleeve environment, and an unpressurised pallet for mounting experiments requiring direct exposure. The complete unit, weighing a maximum of about $14000 \mathrm{~kg}$, will be transported to and from Earth orbit in the payload bay of NASA's shuttle orbiter, and will remain attached to, and supported by, the orbiter throughout each mission. It will be designed for an operational lifetime of 10 years (50 missions, each of seven day's duration, with ground refurbishment). Its major advantages are its reusability and the fact that scientists and engineers (in average-sized crews of four) will be able to go along on the flight to control the experiments and subsystems, and bring their data back with them.

The second of the newly approved projects, the LIIIS launcher, is in- tended to provide Europe, by 1980 , with an independent capability for putting geostationary satellites into orbit. The three-stage launcher will be capable of placing in transfer orbit payloads of $1500 \mathrm{~kg}$, enabling the injection of satellites of some $750 \mathrm{~kg}$ into geostationary orbits with the aid of a suitable apogee motor. The project was put forward by France, following termination of the Europa-ill programme, and the French national space agency, CNES, has already started work on certain parts of the definition phase which will be completed by the end of 1973 . Until the establishment of the European Space Agency, ESRO will liaise between its Member States participating in the LIII S programme and CNES.

The third project, MAROTS (Maritime Orbital Test Satellite), has been generated by the considerable growth of interest in maritime satellites in the last two years. In the U.K., a Geostationary Technology Satellite
(GTS) programme, originally intended for telecommunication purposes, was re-oriented to meet requirements for aiding maritime navigation, and in April 1973 the U.K. proposed that the programme should be Europeanised, taking into account the stage reached at national level. In the meantime, ESRO had been studying the possibility of adapting the telecommunications OTS vehicle for use in a maritime role (the MAROTS proposal). At the European Space Conference meeting on 31 July 1973, the Ministers agreed on the MAROTS proposal, and details of the programme management, etc., are now being worked out.

It is worth noting that each of the three major contributors has undertaken to provide more than half of the financial backing for one of the three new programmes (Germany $52.55 \%$ for Spacelab, France $62.50 \%$ for the LIII S, and the U.K. $56 \%$ for MAROTS).

\title{
Nobel Prize in Physics 1973
}

\section{H. R. Zeller, BBC, Baden}

This year's Nobel prize for physics was awarded to Leo Esaki (IBM, USA), Ivar Giaever (General Electric, USA) and Brian Josephson (Cambridge, England) for their pioneering work in the field of electron tunneling.

In the early days of quantum mechanics, it was shown that a particle can pass across a potential barrier even if classically its energy is insufficient. This effect became known as the tunnel-effect and the related theory was developed by Gamov in connection with $\alpha$-decay where the $\alpha$-particles tunnel across the Coulomb barrier of the nucleus. In the late fifties, Esaki and Giaever introduced tunneling into solid state physics.

The tunnel diode invented by Esaki consists of a p-n junction in which both $\mathrm{n}$ and $\mathrm{p}$ side are heavily doped to make the material degenerate and the transition sharp. For small voltages the electrons in the conduction band of the n-material directly tunnel into the empty states of the valence band of the p-side. At higher voltages no energy conserving tunneling pro- cesses are possible due to the lack of empty states on the opposite side of the junction. This results in a negative resistance region. It is this negative resistance region on which the technical applications of the Esaki diode are based. By slightly oxidizing a metal strip and subsequent vapour deposition of a cross strip, Giaever produced a sandwich consisting of two metal films separated by a thin (20 - 50 A) oxide barrier. Tunneling takes place through the oxide barrier and results in a small-voltage conductivity of the order of $1 \Omega \mathrm{mm}^{-2}$ of the oxide.

The first scientific success of the new technique was Giaever's demonstration of the validity of the BardeenCooper-Schrieffer (BCS) theory of superconductivity. Both the existence of an energy gap and the particular density of states predicted by the BCS theory were experimentally verified.

The tremendous success of tunneling in superconductivity suggested that it could be used more generally as a probe to study all sorts of excitations of the solid state. Subsequent work showed in fact that it was possible to observe molecular vibrations, phonons, plasmons, magnetic excitations, etc., in a very simple form of spectroscopy by voltmeter. But it also became evident that, with the exception of superconductivity, tunneling is mainly probing surface and not bulk properties.

Although the Josephson effect is not necessarily related to tunneling, it was first experimentally observed in tunnel junctions. Josephson showed that if two superconductors are weakly coupled, for instance by the tunnel effect in a superconductorinsulator-superconductor sandwich, there will be a phase relation between the two superconductors. In other words, the whole structure may behave as one superconductor with a supercurrent flowing through the oxide. Generally speaking, the Josephson effect is an example of a macros- 


\section{THE PHYSICIST'S CONCEPTION OF NATURE \\ Edited by JAGDISH MEHRA}

Accounts of the principal conceptions of modern physics, in historical perspective, by some of their most distinguished creators and exponents - an international symposium on "The Development of the Physicist's Conception of Nature in the Twentieth Century". held in Miramare, Trieste, Italy, 18-25 September 1972.

Contents: Space, Time and Geometry: Contributions by D. W. Sciama, S. Chandrasekhar, P. A. M. Dirac, P. Jordan, J. Ehlers, J. Mehra, A. Trautman J. A. Wheeler. - Quantum Theory: Contributions by L. Rosenfeld, W. Heisenberg, B. L. van der Waerden, P. Jordan, J. M. Jauch, E. P. Wigner, F. Rohrlich, R. E. Peierls, G. Wentzel, S. Tomonaga J. Schwinger, A. Salam, C. N Yang, V. G. Telegdi, H. B. G. Casimir. - Statistical Description of Nature: Contributions by G. E. Uhlenbeck, M. Kac, W. E. Lamb Jr., E. G. D. Cohen, I. Prigogine M. Eigen. - Physical Description. Epistemology and Philosophy Contributions by C. F. von Weizsäcker, L. N. Cooper, J. M. Jauch, J. S. Bell, R. Haag, I. Prigogine, G. Ludwig, D. Finkelstein, B. d'Espagnat, C. F. von Weizsäcker.

Cloth, approx. 820 pages

Price: Dfl. 200,-/US\$ 75.00 (approx.)

"This symposium brought back the past and put the great achievements of physics in clearer perspective than any conference I have ever attended. What a marvellous collection of speakers and topics!"

John Archibald Wheeler (Princeton University)

D. REIDEL PUBLISHING COMPANY

Dordrecht-Holland/

Boston-U.S.A.

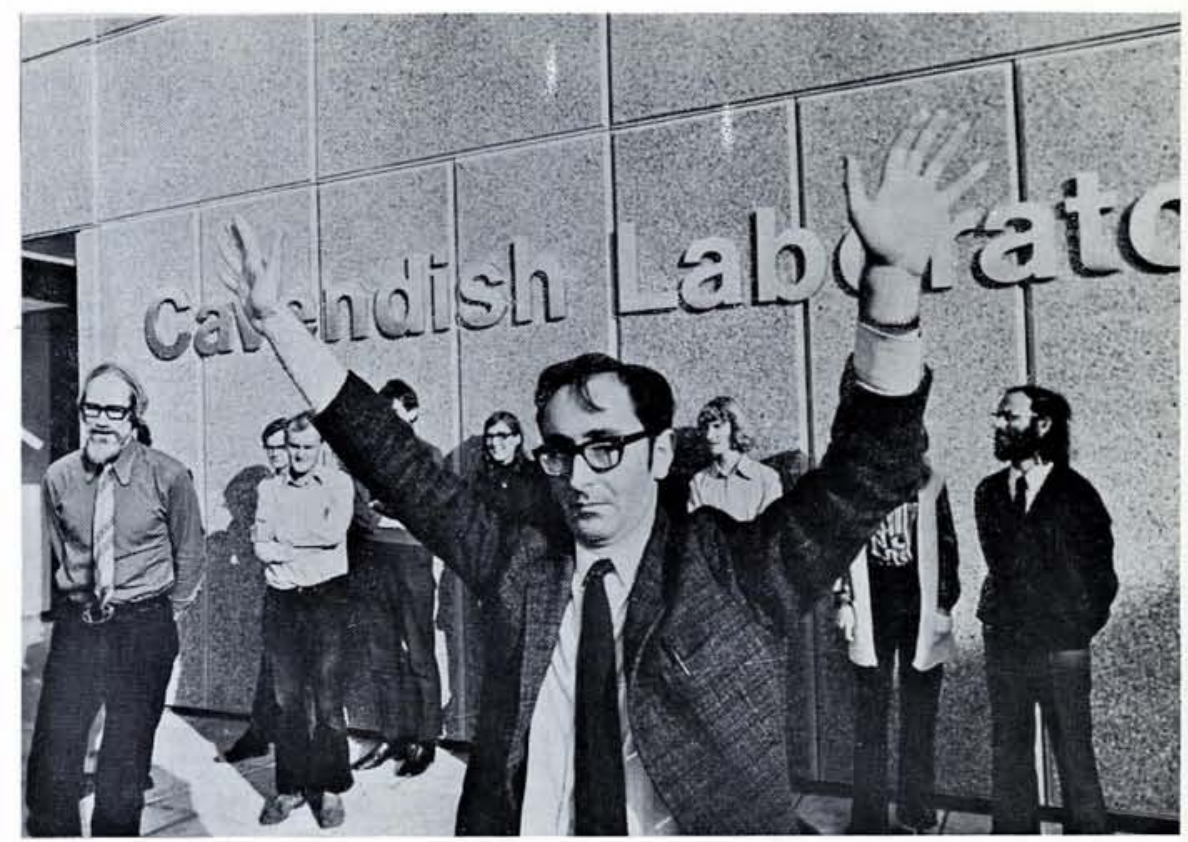

B.D. Josephson celebrates his Nobel Prize award at the University of Cambridge

copic quantum effect. At finite voltages, $V$, electromagnetic radiation will be generated according to the relation $\mathrm{hv}=2 \mathrm{eV}$. The proportionality factor $\frac{2 \mathrm{e}}{\mathrm{h}} \sim 500 \mathrm{MHz} \mu \mathrm{V}^{-1}$

contains only fundamental constants.
Based on the above relation it was possible to determine $\frac{h}{e}$ with high accuracy and to create a new voltage standard by reducing a voltage measurement to a more simple frequency measurement.

\title{
The Physics of the Rare Earth Metals
}

\author{
Elsinore, Denmark, 29 August - 1 September 1973
}

A Europhysics Study Conference on the Rare Earth Metals was held at the LO-Højskole, Elsinore, Denmark, in the period 29 August-1 September, 1973, immediately following the International Magnetism Conference in Moscow. The conference was sponsored by the Metals and Magnetism Sections of EPS and by the Danish Atomic Energy Commission. The National Science Foundation provided partial travel support for some participants from the U.S.A.

The LO-Højskole is an educational centre belonging to the Danish Trades Unions, and it is situated by the sea, in very pleasant surroundings, about 25 miles from Copenhagen. In order to restrict the number of participants to approximately 100 , it was necessary to reject a number of applications to attend the conference. It was a truly international meeting, with participants from 16 different countries. The summary session was held at the Danish AEC Research Establishment Risø, after which the participants had an opportunity of seeing the establishment, especially the neutron scattering facilities.

The philosophy and organization of the conference followed broadly that of the Gordon Conferences, with emphasis on informal discussions, and no published proceedings. The aim was to review the present experimental and theoretical understanding of the physics of the rare earth metals, and this was accomplished principally by means of invited half-hour review papers and the resulting discussions, led by the session chairman. There was also the opportunity to present and discuss short contri- 